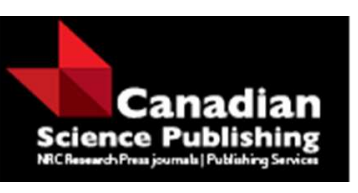

Canadian Journal of Physics

Revue canadienne de physique

\title{
Determination of effective atomic numbers and electron densities from mass attenuation coefficients for some selected complexes containing lanthanides
}

\begin{tabular}{|r|l|}
\hline Journal: & Canadian Journal of Physics \\
\hline Manuscript ID & cjp-2016-0811.R1 \\
\hline Manuscript Type: & Article \\
\hline Complete List of Authors: & $\begin{array}{l}\text { Akman, Ferdi; Bingöl University, Department of Electronic Communication } \\
\text { Technology } \\
\text { Kaçal, M.R.; Giresun Univ, } \\
\text { Akman, Feride; Bingol University, Vocational School of Technical Sciences, } \\
\text { Department of Vegetable and Animal Production } \\
\text { Soylu, Mustafa; Giresun Universitesi, Physics }\end{array}$ \\
\hline Keyword: & $\begin{array}{l}\text { mass attenuation coefficient, effective atomic number, effective electron } \\
\text { density, complex, lanthanide }\end{array}$ \\
\hline $\begin{array}{r}\text { Is the invited manuscript for } \\
\text { consideration in a Special } \\
\text { Issue? : }\end{array}$ & N/A \\
\hline &
\end{tabular}

SCHOLARONE ${ }^{m}$

Manuscripts 


\title{
Determination of effective atomic numbers and electron densities from mass attenuation coefficients for some selected complexes containing lanthanides
}

\author{
Ferdi Akman, ${ }^{\mathrm{a},}$, Mustafa Recep Kaçal ${ }^{\mathrm{b}}$, Feride $\mathrm{Akman}^{\mathrm{c}}$, Mustafa Serkan Soylu ${ }^{\mathrm{b}}$

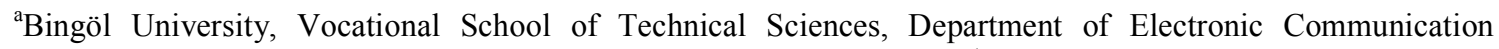 \\ Technology, $12000 \quad$ Bingöl, Turkey. Fax:+904262150788 ${ }^{\text {; }} \quad$ Tel:+904262160012-4067; E- \\ mail:fakman@bingol.edu.tr \\ ${ }^{\mathrm{b}}$ Giresun University, Sciences and Arts Faculty, Department of Physics, 28100 Giresun, Turkey. \\ 'Bingöl University, Vocational School of Technical Sciences, Department of Vegetable and Animal Production, \\ 12000, Bingöl, Turkey.
}

\begin{abstract}
The effective atomic numbers and electron densities for the pure elemental forms of Gadolinium (Gd), Dysprosium (Dy), Erbium (Er), and Ytterbium (Yb) and their some selected complexes were obtained from the measured total mass attenuation coefficients at 13.92, 17.75, 20.78, 26.34 and $59.54 \mathrm{keV}$ photon energies using a high-resolution $\mathrm{Si}(\mathrm{Li})$ detector by adopting transmission geometry. The measured results were compared with the two different theoretical results. Within experimental deviations, our data are in good agreement with the theoretical values. The obtained parameters were also interpreted with some selected chemical parameters.
\end{abstract}

Keywords: Mass attenuation coefficient; effective atomic number; electron density; complex, lanthanide.

\footnotetext{
${ }^{*}$ Corresponding author. Tel:+ 904262160012-1331

Fax: +904262150788

E-mail addresses: fakman@bingol.edu.tr

(F. Akman)
} 


\section{Introduction}

Lanthanides and their complexes are useful in technology and industry. Lanthanide ion complexes are specially used in medical applications because of their unique luminescence properties. They have large Stokes shifts and extremely long emission lifetimes. Lanthanide (III) complexes are used as contrast agents in Magnetic Resonance Imaging diagnostic techniques and shift reagents in Nuclear Magnetic Resonance analyses of electrolytes in biological tissues.

The most used parameter in the photon interaction with matter is total mass attenuation coefficient which is the sum of absorption and scattering coefficients. The total mass attenuation coefficient is a measure of the probability of the interaction that occurs between incident photons and the material of the unit mass per unit area. This parameter depends on the atomic number of sample, the sample density, the incident photon energy, and the chemical structure of the sample. The total mass attenuation coefficient is an essential tool to obtain many other parameters like atomic, molecular and electronic cross sections, effective atomic number, effective electron density, kinetic energy released per unit mass (Kerma), and molar extinction coefficient. The measurement or calculation of these parameters is a pioneer attempt for many applied fields. These parameters are widely used in medical, radiation, nuclear, plasma, and space physics, biological and agricultural industries, so accurate data of these parameters have a great importance.

A single atomic number is utilized to describe an element. According to Hine [1], the practical applications of photon interaction with the complex material can be defined by using an effective atomic number, $Z_{\text {eff, }}$ and the $Z_{\text {eff }}$ varies with energy. For this reason, $Z_{\text {eff }}$ cannot be indicated uniquely across the entire region by a single number like in the case of single elements. For each of different process by which photons can interact with matter, the various atomic number in the material have to be weighted differently. Similarly, the effective electron density indicates the interaction characteristic with a complex material and it is defined as the number of electrons per unit mass of the material. The effective atomic number and electron density are used in the determination of Kerma, mass energy absorption coefficients, and Compton profiles of complex materials. The effective atomic number and electron density ensure knowledge of complex for photon interaction within the mixtures of materials.

Many researchers or research groups determined the mass attenuation coefficients, effective atomic numbers and electron densities of composite materials like compounds, complexes, glasses, polymers and biological materials [2-21]. Some of those; El-Kateb and Abdul-Hamid [2] estimated that the mass attenuation coefficients, effective atomic numbers and electron densities in materials containing $\mathrm{H}, \mathrm{C}$, and $\mathrm{O}$ in the energy range 0.054-1.333 $\mathrm{MeV}$ using a $3 \mathrm{X} 3$ inch $\mathrm{NaI}(\mathrm{Tl})$ scintillation detectors. Gill et al. [5] obtained the effective atomic numbers of some glasses such as $\mathrm{PbO}, \mathrm{CdO}, \mathrm{Bi}_{2} \mathrm{O}_{3}, \mathrm{~B}_{2} \mathrm{O}_{3}$ and rocks such as granite, rhyolite, migmatite, phosphorite, and limestone at the $662 \mathrm{keV}$ energy using a $3.81 \mathrm{~cm} \mathrm{X} 3.81$ $\mathrm{cm} \mathrm{NaI(Tl)} \mathrm{scintillation} \mathrm{detector.} \mathrm{The} \mathrm{total} \mathrm{mass} \mathrm{attenuation} \mathrm{coefficients,} \mathrm{total} \mathrm{atomic} \mathrm{and}$ electronic cross sections, effective atomic numbers and electron densities of undoped and 
differently doped GaAs and InP crystals were estimated by Demir and Han [7]. Medhat [11] measured the total mass attenuation coefficients, total atomic and electronic cross sections, effective atomic numbers and electron densities of some gemstones at 81, 356.5, 661.6, 1173.2 and $1332.5 \mathrm{keV}$ photon energies using an HPGe detector. The mass attenuation coefficients effective atomic numbers and electron densities of some selected amino acids were measured by Kore and Pawar [15] at 122, 356, 511, 662, 1170, 1275 and $1330 \mathrm{keV}$ photon energies with the help of a $\mathrm{NaI}(\mathrm{Tl})$ detector. The $\mathrm{K}$ shell absorption jump ratios, jump factors, effective atomic numbers and electron densities from the measured total mass attenuation coefficients for the some selected compounds of gadolinium in the energy range from 39.52 to $57.14 \mathrm{keV}$ energies were obtained by Akman et al. [20] and the results were interpreted with the help of some chemical parameters such as energy gap, ionization energy, electron affinity, and global electrophilicity of ligands.

In this study, the mass attenuation coefficients, effective atomic numbers and electron densities for the pure elemental forms of Gd, Dy, Er and $\mathrm{Yb}$ and the obtained some complexes forms of these elements were determined at 13.92, 17.75, 20.78, 26.34 and $59.54 \mathrm{keV}$ photon energies by adopting transmission geometry. The results were compared with the two different theoretical results. To interpret the measured results, we calculated the energy gap, ionization energy, electron affinity, chemical hardness, chemical softness, chemical potential, electronegativity and global electrophilicity of malonic and phenylmalonic acids using the Density Functional Theory/Becke-3-Lee-Yang-Parr (DFT/B3LYP) method supplemental with SDD basis set.

\section{Experimental process and data analysis 2.1. Sample preparation}

In the synthesis of $\mathrm{X}(\mathrm{III}) \mathrm{Cl}(\mathrm{PhMal}) .5 \mathrm{H}_{2} \mathrm{O}(\mathrm{X}=\mathrm{Gd}, \mathrm{Dy}, \mathrm{Er}$ and $\mathrm{Yb})$ complex, a solution of phenylmalonic acid $\left(\mathrm{PhMalH}_{2}\right)(0.360 \mathrm{~g}, 2 \mathrm{mmol})$ and $\mathrm{NaOH}(0.159 \mathrm{~g}, 4 \mathrm{mmol})$ in water $(25$ $\mathrm{mL}$ ) was added dropwise with stirring at $60^{\circ} \mathrm{C}$ to a solution of $\mathrm{X}(\mathrm{III})$ chloride hexahydrate $\left(\mathrm{XCl}_{3} .6 \mathrm{H}_{2} \mathrm{O}\right)(0.743 \mathrm{~g}$ for $\mathrm{Gd}, 0.753 \mathrm{~g}$ for $\mathrm{Dy}, 0.763 \mathrm{~g}$ for $\mathrm{Er}$ and $0.774 \mathrm{~g}$ for $\mathrm{Yb})(2 \mathrm{mmol})$ in distilled water and ethanol solution $(25 \mathrm{~mL})$. The mixture was stirred for $6 \mathrm{~h}$ at $60{ }^{\circ} \mathrm{C}$ and then cooled to room temperature. The formed crystals were filtered and washed with $10 \mathrm{~mL}$ of water and were dried in air. The chemical reaction equation of $\mathrm{X}(\mathrm{III}) \mathrm{Cl}(\mathrm{PhMal}) \cdot 5 \mathrm{H}_{2} \mathrm{O}$ is given as follows,

$$
\mathrm{PhMalH}_{2}+\mathrm{X}(\mathrm{III}) \mathrm{Cl}_{3} \cdot 6 \mathrm{H}_{2} \mathrm{O}+2 \cdot(\mathrm{NaOH}) \rightarrow \mathrm{X}(\mathrm{III}) \mathrm{Cl}(\mathrm{PhMal}) \cdot 5 \mathrm{H}_{2} \mathrm{O}+3 \mathrm{H}_{2} \mathrm{O}
$$

In the synthesis of $\mathrm{X}(\mathrm{III}) \mathrm{Cl}(\mathrm{Mal}) .5 \mathrm{H}_{2} \mathrm{O}(\mathrm{X}=\mathrm{Gd}$, Dy, Er and $\mathrm{Yb})$ complex, a solution of malonic acid $\left(\mathrm{MalH}_{2}\right)(0.208 \mathrm{~g}, 2 \mathrm{mmol})$ and $\mathrm{NaOH}(0.159 \mathrm{~g}, 4 \mathrm{mmol})$ in water $(25 \mathrm{~mL})$ was added dropwise with stirring at $60{ }^{\circ} \mathrm{C}$ to a solution of $\mathrm{X}(\mathrm{III})$ chloride hexahydrate $\left(\mathrm{XCl}_{3} \cdot 6 \mathrm{H}_{2} \mathrm{O}\right)$ ( $0.743 \mathrm{~g}$ for $\mathrm{Gd}, 0.753 \mathrm{~g}$ for $\mathrm{Dy}, 0.763 \mathrm{~g}$ for $\mathrm{Er}$ and $0.774 \mathrm{~g}$ for $\mathrm{Yb})(2 \mathrm{mmol})$ in distilled water and ethanol solution $(25 \mathrm{~mL})$. The mixture was stirred for $6 \mathrm{~h}$ at $60{ }^{\circ} \mathrm{C}$ and then cooled to room temperature. The formed crystals were filtered and washed with $10 \mathrm{~mL}$ of water and were dried in air. The chemical reaction equation of $\mathrm{X}(\mathrm{III}) \mathrm{Cl}(\mathrm{Mal}) .5 \mathrm{H}_{2} \mathrm{O}$ is given as follows,

$$
\mathrm{MalH}_{2}+\mathrm{X}(\mathrm{III}) \mathrm{Cl}_{3} \cdot 6 \mathrm{H}_{2} \mathrm{O}+2 \cdot(\mathrm{NaOH}) \rightarrow \mathrm{X}(\mathrm{III}) \mathrm{Cl}(\mathrm{Mal}) \cdot 5 \mathrm{H}_{2} \mathrm{O}+3 \mathrm{H}_{2} \mathrm{O}
$$


The chemical structures of $\mathrm{X}(\mathrm{III}) \mathrm{Cl}(\mathrm{PhMal}) .5 \mathrm{H}_{2} \mathrm{O}$ and $\mathrm{X}(\mathrm{III}) \mathrm{Cl}(\mathrm{Mal}) .5 \mathrm{H}_{2} \mathrm{O}$ are given in Figs. $1 \mathrm{a}$ and $1 \mathrm{~b}$.

The obtained powder samples were passed through a $37 \mu \mathrm{m}$ sieve. The masses of the sieved powder samples were weighed with an electrical balance having a sensitive $0.001 \mathrm{~g}$. This process was repeated five times to determine consistent value of the mass. Then, the powder samples were compressed into disc pellets with a $20 \mathrm{~mm}$ diameter for $20 \mathrm{~s}$ at $2.10^{3}$ $\mathrm{Ncm}^{-2}$ pressures using a manual press machine. The mass per unit area of the samples were determined ranging from $0.020-0.473 \mathrm{gcm}^{-2}$ by using the radius of the pellet and the mass value of sample.

\subsection{Experimental process}

In the present study, the experimental arrangement which is known as transmission geometry is shown schematically in Fig. 2. It consists of a calibrated $\mathrm{Si}(\mathrm{Li})$ detector system, an ${ }^{241} \mathrm{Am}$ annular source, target, and collimators. This setup provides the required good geometry condition for the determination of absorption parameters. A Si(Li) detector which has $8 \mu \mathrm{m}$ thickness Be window, $5 \mathrm{~mm}$ sensitive depth, $12.5 \mathrm{~mm}^{2}$ active area, and $160 \mathrm{eV}$ at $5.9 \mathrm{keV}$ resolution was used to record the intensities of X-and $\gamma$-rays. This detector was coupled with Ortec made $2 \mathrm{~K}$ DSPEC-LF multi-channel analyzer (MCA) system which consists of the high voltage power supply, an amplifier, and an analog digital converter interfaced with a personal computer supplied with Maestro software program for storage and analysis data. To minimize the low energy background and intensity of scattered photons from surrounding, the detector was placed in sandwiching collimator which was made from $\mathrm{Pb}(4.2 \mathrm{~mm}), \mathrm{Fe}(1.1 \mathrm{~mm})$, and Al $(1.0 \mathrm{~mm})$. As seen from Fig.2, the lead collimators of $8 \mathrm{~mm}$ and $5 \mathrm{~mm}$ diameter and $1 \mathrm{~cm}$ thickness were used to collimate the radiation rays. The lead collimator contains the sufficient iron and aluminum for absorbing the L X-rays of lead and the K X-rays of iron, respectively. The energy calibration of detector system was made with the aid of the radioactive standards in the energy range from 0 to $88 \mathrm{keV}$. The absorber samples were placed one by one between the point source and the detector. The distances between the point source with the absorber and the absorber with Be window of the detector were measured equally $14 \mathrm{~cm}$ and $1 \mathrm{~cm}$, respectively. The peak position of $\mathrm{X}$ - and $\gamma$-rays photon energies were controlled before and after the experiments.

An annular ${ }^{241} \mathrm{Am}$ radioactive source which has $3.7 \mathrm{GBq}(100 \mathrm{mCi})$ activity was used as the photon source. This source emits useful intensities at energies of 13.92, 17.75, 20.78, 26.34, and $59.54 \mathrm{keV}$. The 13.92, 17.75, and $20.78 \mathrm{keV}$ energies are the $\mathrm{Np} \mathrm{L} \alpha, \mathrm{L} \beta$, and $\mathrm{L} \gamma$ characteristic X-rays, respectively. The other energies are the gamma-rays which occur after the alpha decay. The emission probabilities of 13.92, 17.75, 20.78, 26.34, and $59.54 \mathrm{keV}$ photon energies are about $9 \%, 6 \%, 1.5 \%, 2.4 \%$, and $35.9 \%$, respectively. The annular source was housed in a disc container made of the lead having a front hole $2 \mathrm{~mm}$ in diameter.

To decrease the statistical uncertainty, the samples were measured by collecting the spectra from the selected complexes for a period of 7200 and thus, the pulse pile-up effects were kept to a minimum. The net counts without absorber and with absorber were performed in fixed time and under the same experimental situations. Although there was small dead time 
loss, we utilized the live time of a MCA which turns out to be real time. The peak areas were determined by using Microcal Origin 7.5 software program (demo) with the least-squares fit method. The area uncertainties were estimated utilizing a weighted method. The peak areas were separated by fitting the obtained spectra with a multi-Gaussian function plus linear backgrounds. The net peak areas were determined from the measured peak area by subtracting the background count. A typical spectrum of related energies transmission through $\mathrm{Er}(\mathrm{III}) \mathrm{Cl}(\mathrm{Mal}) .5 \mathrm{H}_{2} \mathrm{O}$ is presented in Fig. 3.

\subsection{Determination of absorption parameters}

The attenuation of radiation throughout the certain material is expressed by Beer-Lambert law. According to this law, there is a relationship between incoming and absorbed radiation as follows,

$I=I_{0} e^{-\left(\frac{\mu}{\rho}\right) \rho x}$

where $I$ and $I_{0}$ are attenuated and unattenuated radiation intensities, $\mu / \rho$ is the mass attenuation coefficient, $\rho$ is the density of the material and $\mathrm{x}$ is the thickness of material. The mass attenuation coefficient is derived using the Eq. (1) as follows,

$\mu / \rho=-\frac{1}{\rho x} \ln \left(\frac{I}{I_{0}}\right)$

and $\rho x$ is determined using the following equation,

$\rho x=m / \pi r^{2}$

where $\mathrm{m}$ is the mass of the material and $\mathrm{r}$ is the radius of the material in the form of the pellet. The mass attenuation coefficient for materials consisting of multiple elements is determined using the following equation,

$(\mu / \rho)_{\text {comp. }}=\sum_{i} W_{i}(\mu / \rho)_{i}$

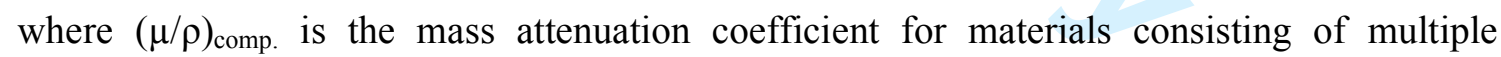
elements, $W_{i}$ is the weight fraction of the ith consistent element in the material and $(\mu / \rho)_{i}$ is the mass attenuation coefficient of the ith consistent element in the material. The weight fraction of a material consisting of multiple elements is obtained the following equation,

$$
W_{i}=\frac{a_{i} A_{i}}{\sum_{j} a_{j} A_{j}}
$$

where $a_{i}$ is the number of atoms of the ith constituent element in the complex material and $A_{i}$ is the atomic weight of the ith consistent element in the complex material.

The values of mass attenuation coefficient can be used to determine a set of absorption coefficients such as molecular, atomic and electronic cross sections, effective atomic number 
and electron density. The mass attenuation coefficient is proportional with the molecular cross section $\left(\sigma_{t, m}\right)$ through the following equation,

$\sigma_{t, m}=\frac{1}{N}(\mu / \rho)_{\text {comp. }} \sum_{i}\left(n_{i} A_{i}\right) \quad\left(\right.$ barn molecule $\left.{ }^{-1}\right)$

where $\mathrm{N}$ is the Avogadro's number, $(\mu / \rho)_{\text {comp. }}$ is the mass attenuation coefficient of the material, $\Sigma_{i}\left(n_{i} A_{i}\right)$ is the molecular weight of the material, $n_{i}$ is the number of atoms of the ith consistent element and $A_{i}$ is its atomic weight. The atomic cross section $\left(\sigma_{t, a}\right)$ is obtained with the aid of molecular cross section as follows,

$\sigma_{t, a}=\sigma_{t, m} \frac{1}{\sum_{i} n_{i}} \quad\left(\right.$ barn atom $\left.{ }^{-1}\right)$

where $\Sigma_{\mathrm{i}} \mathrm{n}_{\mathrm{i}}$ is the total number of atoms in the material. The electronic cross section $\left(\sigma_{\mathrm{t}, \mathrm{e}}\right)$ for the individual element in the material is determined using the following equation,

$\sigma_{t, e}=\frac{1}{N} \sum_{i} \frac{f_{i} A_{i}}{Z_{i}}(\mu / \rho)_{i} \quad\left(\right.$ barn electron $\left.^{-1}\right)$

where, $A_{i}$ and $Z_{i}$ are the atomic weight and the atomic number of ith consistent element in the material, $(\mu / \rho)_{\mathrm{i}}$ is the mass attenuation coefficient of ith consistent element and $\mathrm{f}_{\mathrm{i}}$ is the fractional abundance of the ith element in the material. The fractional abundance of an element in the material is obtained from $n_{i} / \Sigma n_{i}$ where $n_{i}$ is the number of atoms of the ith consistent element and $\Sigma \mathrm{n}_{\mathrm{i}}$ is the total number of atoms in the material.

Using the atomic and electronic cross sections, the effective atomic number $\left(Z_{\text {eff }}\right)$ can be estimated as follows,

$Z_{e f f}=\frac{\sigma_{t, a}}{\sigma_{t, e}} \quad$ (dimensionless quantity)

namely, the effective atomic number is the ratio of atomic cross section to electronic cross section. Finally, the electron density is determined using the effective atomic number as follows,

$N_{E}=\frac{Z_{\text {eff }}}{A_{\text {tot }}}\left(N n_{\text {tot }}\right) \quad\left(\right.$ electrons $\left.g^{-1}\right)$

here, $\mathrm{Z}_{\mathrm{eff}}$ is the effective atomic number, $\mathrm{A}_{\text {tot }}$ is the molecular weight of the material, $\mathrm{N}$ is the Avogadro's number and $\mathrm{n}_{\text {tot }}$ is the total number of atoms in the material.

\subsection{Calculation of chemical parameters}

To interpret the experimental results of absorption parameters, some chemical parameters such as energy gap $(\Delta \mathrm{E})$, ionization energy $(\mathrm{I})$, electron affinity $(\mathrm{A})$, electronegativity $(\chi)$, chemical hardness $(\eta)$, chemical softness (S), chemical potential $(\mu)$ and global electrophilicity $(\omega)$ of malonic and phenylmalonic acids were calculated with the help of the 
highest occupied molecular orbital (HOMO) energy and lowest unoccupied molecular orbital (LUMO) energy. The HOMO and LUMO energies were calculated with the Windows version of Gaussian 09 package program [22] and Gaussview 5.0.9 molecular visualization program [23] using Density Functional Theory/Becke-3-Lee-Yang-Parr (DFT/B3LYP) method supplemental with SDD basis set. HOMO behaves as electron donor while LUMO behaves as acceptor. The difference between HOMO and LUMO energies presents the HOMO-LUMO energy gap. If a molecule has a small energy gap, it shows a high chemical reactivity. The ionization energy and electron affinity parameters were determined using the energies of HOMO and LUMO, namely, $\mathrm{I}=-\mathrm{E}_{\mathrm{HOMO}}$ and $\mathrm{A}=-\mathrm{E}_{\mathrm{LUMO}}$ where $\mathrm{I}$ is the ionization energy and $\mathrm{A}$ is the electron affinity. The chemical hardness and softness of a molecule are qualitative indications, how much its electron cloud is distorted in an electric field and they can be determined as following equations;

$$
\begin{aligned}
& \eta=\frac{E_{L U M O}-E_{\text {НОМO }}}{2}=\frac{I-A}{2} \\
& S=\frac{1}{\eta}=\frac{2}{E_{L U M O}-E_{\text {HOMO }}}=\frac{2}{I-A}
\end{aligned}
$$

Electronegativity is the tendency of molecules to attract electrons and it can be determined as follows;

$\chi=-\left(\frac{E_{\text {номо }}-E_{L U M O}}{2}\right)=\frac{I+A}{2}$

The chemical potential relates to charge transfer from a system of higher chemical potential to lower chemical potential. It presents the affinity of an electron to flee and it can be determined as follows;

$$
\mu=\left(\frac{E_{\text {Номо }}-E_{\text {LUMO }}}{2}\right)=-\left(\frac{I+A}{2}\right)
$$

The capability of a substance to accept electrons is quantified as electrophilicity index. It measures the energy lowering of a substance due to the electron flow between donor and acceptor. It can be obtained using the following equation;

$$
\omega=\frac{\mu^{2}}{2 \eta}=\left\{\frac{\left(\frac{E_{H O M O}-E_{L U M O}}{2}\right)^{2}}{E_{L U M O}-E_{\text {HOMO }}}\right\}=\left\{\frac{\left(\frac{I+A}{2}\right)^{2}}{I-A}\right\}
$$

The calculated chemical parameters are listed in Table 1.

\section{Results and discussion}


The total mass attenuation coefficients $(\mu / \rho)$, effective atomic numbers $\left(Z_{\text {eff }}\right)$ and electron densities $\left(\mathrm{N}_{\mathrm{E}}\right)$ of $\mathrm{Gd}$, Dy, Er, $\mathrm{Yb}$ and their selected complexes were determined using the narrow beam transmission geometry at 13.92, 17.75, 20.78, 26.34 and $59.54 \mathrm{keV}$ photon energies. The maximum uncertainty in the measured experimental parameters is estimated to be $5.85 \%$. This uncertainty is attributed to the areas under the related $\mathrm{X}$ - and $\gamma$-ray peaks, mass per unit area of the sample, statistical uncertainties in the incident (I) and transmitted ( $\left.\mathrm{I}_{0}\right)$ and systematic uncertainties. The maximum uncertainty in total mass attenuation coefficient was determined from uncertainties in incident $\left(\mathrm{I}_{0}\right)$, and transmitted (I) intensities and mass per unit area (t) using the propagation of uncertainty formula [24],

$\Delta(\mu / \rho)=\frac{1}{t} \sqrt{\left(\frac{\Delta I_{0}}{I_{0}}\right)^{2}+\left(\frac{\Delta I}{I}\right)^{2}+\left(\ln \frac{I_{0}}{I}\right)^{2}\left(\frac{\Delta t}{t}\right)^{2}}$

where, $\Delta \mathrm{I}_{0}$ and $\Delta \mathrm{I}$ are the uncertainties of $\mathrm{I}_{0}$ and, $\mathrm{I}$ and $\Delta \mathrm{t}$ is the uncertainty of mass per unit area. The experimental values of $\mu / \rho$ were obtained using the incident intensity of $X$ - and $\gamma$ rays, transmitted intensity of $\mathrm{X}$ - and $\gamma$-rays and mass per unit area of the sample. The experimental values of $\mu / \rho$ at $13.92,17.75,20.78,26.34$ and $59.54 \mathrm{keV}$ photon energies are listed in Table 2. This table also includes the WinXCOM [25] values of $\mu / \rho$, which calculates based on the Hartree-Slater central potential theory and the FFAST [26] values of $\mu / \rho$, which calculates based on the self-consistent Dirac-Hartree-Fock theory. The maximum differences between the WinXCOM and FFAST values were found to be $2.57 \%$ for the present parameters and energies. The variation of $\mu / \rho$ for Dy and its selected complexes is plotted as a function of photon energy in Fig. 4. From the Table 2 and Fig. 4, it is evident that the $\mu / \rho$ values decrease exponentially with increasing energy. As seen from Table 2 , the $\mu / \rho$ values decrease with increasing atom number. These variations are due to the dominance of the photoelectric effect which depends on the atomic number as $Z^{4}$ for low energy and $Z^{5}$ for high energy and the photoelectric effect is inversely proportional to energy as $E^{3.5}$. The experimental values of $\mu / \rho$ agreed to be within $0.05 \%-4.68 \%$ of the theoretical values of WinXCOM and $0.05 \%-6.18 \%$ of the theoretical values of FFAST. It is seen from the Table 2 and Fig. 4 that the experimental values of $\mu / \rho$ are in good agreement with theoretical results of WinXCOM and FFAST within the experimental uncertainties. Significant differences due to chemical composition are observed in $\mu / \rho$ as seen in Fig. 4. The $\mu / \rho$ values of Gd, Dy, Er and $\mathrm{Yb}$ at $59.54 \mathrm{keV}$ photon energy differ from their complexes by approximately $\sim 55 \%$, $\sim 64 \%, \sim 63 \%$ and $\sim 59 \%$, respectively.

The effective atomic numbers $\left(Z_{\text {eff }}\right)$ for present samples were determined using the Eq. (9), namely, by the ratio of the atomic cross section and the electronic cross section. The experimental $Z_{\text {eff }}$ values are presented in Table 3 along with the theoretical values. The changes of effective electron densities $\left(\mathrm{N}_{\mathrm{E}}\right)$ show almost similar behavior to $Z_{\text {eff. }}$ The $\mathrm{N}_{\mathrm{E}}$ values are listed in Table 4. Typical graphics of $\mathrm{Dy}(\mathrm{III}) \mathrm{Cl}(\mathrm{PhMal}) .5 \mathrm{H}_{2} \mathrm{O}$ for $\mathrm{Z}_{\mathrm{eff}}$ and $\mathrm{N}_{\mathrm{E}}$ are given in Figs. 5-6, respectively. The experimental values of $\mathrm{Z}_{\mathrm{eff}}$ and $\mathrm{N}_{\mathrm{E}}$ agreed to be within $0 \%-4.67 \%$ of the theoretical values of WinXCOM and $0.03 \%-5.13 \%$ of the theoretical values of FFAST. As seen from Tables 3-4, the $Z_{\text {eff }}$ values for the present samples generally decrease with increasing atom number and photon energy. The $\mathrm{N}_{\mathrm{E}}$ values increase with increasing atom 
number and decrease with increasing photon energy. It is clearly seen from Table 3 that the present data of $Z_{\text {eff }}$ support those of Hine [1]. As shown in Tables 3-4, the variations of $Z_{\text {eff }}$ and $\mathrm{N}_{\mathrm{E}}$ depend on the atom number, photon energy and chemical composition of complexes. Also, it is clearly seen from Tables 3-4 that the $Z_{\text {eff }}$ and $N_{E}$ values change with the number of elements, the range of atomic numbers in a complex and total atomic weight. These variations are attributed to different binding energies and inter-atomic distances depend on different interactions between central atom and ligands in the complexes. In the process of complex formation, some valence electron is transferred from the atom in the complex. Therefore, the electronic screening varies and the binding energies of the outer shell change.

It is clearly seen from Tables $1-4$, the $\mu / \rho, Z_{\text {eff }}$ and $N_{E}$ parameters are varied with energy gap $(\Delta \mathrm{E})$, ionization energy (I), electron affinity (A), hardness $(\eta)$, softness $(S)$, chemical potential $(\mu)$, electronegativity $(\chi)$ and global electrophilicity $(\omega)$ of malonic and phenylmalonic acids. According to the Tables 1-4, the $\mu / \rho$ and $Z_{\text {eff }}$ values of $\mathrm{X}(\mathrm{III}) \mathrm{Cl}(\mathrm{Mal}) .5 \mathrm{H}_{2} \mathrm{O}$ are bigger than that of $\mathrm{X}(\mathrm{III}) \mathrm{Cl}(\mathrm{PhMal}) .5 \mathrm{H}_{2} \mathrm{O}$ values, and the $\mathrm{N}_{\mathrm{E}}$ values of $\mathrm{X}(\mathrm{III}) \mathrm{Cl}(\mathrm{Mal}) .5 \mathrm{H}_{2} \mathrm{O}$ are smaller than that of $\mathrm{X}(\mathrm{III}) \mathrm{Cl}(\mathrm{PhMal}) .5 \mathrm{H}_{2} \mathrm{O}$ values. We can say that the $\mu / \rho$ and $Z_{\text {eff }}$ values increase with increasing $\Delta \mathrm{E}, \mathrm{I}, \eta$ and $\chi$ values and decrease with increasing $\mathrm{A}, \mathrm{S}, \mu$ and $\omega$ values. Furthermore, the $\mathrm{N}_{\mathrm{E}}$ values decrease with increasing $\Delta \mathrm{E}, \mathrm{I}, \eta$ and $\chi$ values and increase with increasing $A, S, \mu$ and $\omega$ values.

\section{Conclusions}

We investigated the variation of the mass attenuation coefficients, effective atomic numbers and electron densities of some selected complexes with photon energy and atom number in a complex. The experiments were performed using a high-resolution $\mathrm{Si}(\mathrm{Li})$ detector at 13.92, $17.75,20.78,26.34$ and $59.54 \mathrm{keV}$ photon energies. The experimental results were compared with two different theoretical results. Also, the changes in the absorption parameters were interpreted with the some selected chemical parameters. The $\mu / \rho, Z_{\text {eff }}$ and $N_{E}$ values depend on photon energy, atom number in a complex and chemical composition of complex materials. It is observed that the present parameters change with total atomic weight and number of elements within complex. Also, the present parameters vary with the chemical parameters such as energy gap, ionization energy, electron affinity, hardness, softness, chemical potential, electronegativity and global electrophilicity of malonic and phenylmalonic acids. The calculated chemical parameters can be used to interpret the absorption parameters. Also, without performing any experimental or theoretical study, they may be used to predict that how the absorption parameters can be changed by the bonded ligand group. Therefore, these chemical parameters can be used as a tool for accurate predictions of absorption parameters.

\section{Acknowledgements}

This work was done with the support of the Bingöl University Research Fund under Project No. BAP-195-300-2015 and the authors wish to thank Bitlis Eren University for supporting the Gaussian 09W and Gaussian 05 view software. 


\section{References}

[1] G.J. Hine. Phys. Rev. 85,725 (1952).

[2] A.H. El-Kateb and A.S. Abdul-Hamid. Appl. Radiat. Isotopes 42, 303 (1991).

[3] G.S. Bhandal and K. Singh. Appl. Radiat. Isotopes 44, 1231 (1993).

[4] G.S. Bhandal and K. Singh. Appl. Radiat. Isotopes 44, 929 (1993).

[5] H. Gill, G. Kaur, K. Singh, V. Kumar, and J. Singh. Radiat. Phys. Chem. 51, 671 (1998).

[6] O. İçelli, S. Erzeneoğlu, and R. Boncukçuoğlu. Nucl. Instrum. Meth. B 266, 3226 (2008).

[7] L. Demir and I. Han. Ann. Nucl. Energy 36, 869 (2009).

[8] I. Han, L. Demir, and M. Şahin. Radiat. Phys. Chem. 78, 760 (2009).

[9] R. Polat, Z. Yalçın, and O. İçelli. Nucl. Instrum. Meth. A 629, 185 (2011).

[10] D. Demir, A. Turşucu, and T. Öznülüer. Radiat. Environ. Bioph. 51, 469 (2012).

[11] M. Medhat. J. Radioanal. Nucl. Ch. 293, 555 (2012).

[12] M. Ahmadi, N. Lunscher, and J.T.W. Yeow. Nucl. Instrum. Meth. B, 300, 30 (2013).

[13] P.P. Pawar and G.K. Bichile. Radiat.Phys. Chem. 92, 22 (2013).

[14] N. Kucuk, M. Cakır, and N.A. Isitman. Radiat. Prot. Dosim. 153, 127 (2013).

[15] P.S. Kore and P.P. Pawar. Radiat. Phys. Chem. 98, 86 (2014).

[16] P. Yasaka, N. Pattanaboonmee, H.J. Kim, P. Limkitjaroen, and J. Kaewkhao. Ann. Nucl. Energy 68, 4 (2014).

[17] F. Akman, R. Durak, M.F. Turhan, and, M.R. Kaçal. Appl. Radiat. Isotopes 101, 107 (2015).

[18] B. Oto, N. Y1ld1z, F. Akdemir, and E. Kavaz. Prog. Nucl. Energ. 85, 391 (2015).

[19] F. Akman, R. Durak, M.R. Kaçal, M.F. Turhan, and F. Akdemir. Radiat. Phys. Chem. 107, 75 (2015).

[20] F. Akman, R. Durak, M.R. Kaçal, and F. Bezgin. X-ray Spectrom. 45, 103 (2016).

[21] C. V. More, R.M. Lokhande, and P.P. Pawar. Radiat. Phys. Chem. 125, 14 (2016).

[22] M.J. Frisch, R.A.G.W. Trucks, H.B. Schlegel et al. Gaussian 09, Gaussian Inc. Wallingford C.T. (2010).

[23] R. Dennington, T. Keith, and J. Millam. Gauss. View Version 5, Semichem. Inc. Shawnee, Mission K.S. (2010).

[24] F. Akman, R. Durak, and M.R. Kaçal. Can. J. Phys. 93, 1532 (2015).

[25] L. Gerward, N. Guilbert, K.B. Jensen, and H. Levring. Radiat. Phys. Chem. 60, 23 (2001). 
[26] C.T. Chantler. J. Phys.Chem. Ref. Data 29, 597 (2000). 


\section{Figure Captions}

Fig. 1. (a, b) The chemical structures of $\mathrm{X}(\mathrm{III}) \mathrm{Cl}(\mathrm{PhMal}) \cdot 5 \mathrm{H}_{2} \mathrm{O}$ and $\mathrm{X}(\mathrm{III}) \mathrm{Cl}(\mathrm{Mal}) \cdot 5 \mathrm{H}_{2} \mathrm{O}$

Fig. 2. Experimental set-up

Fig. 3. A typical spectrum of related energies transmission through $\mathrm{Er}(\mathrm{III}) \mathrm{Cl}(\mathrm{Mal}) .5 \mathrm{H}_{2} \mathrm{O}$

Fig. 4. Mass attenuation coefficient versus photon energy for Dy and its selected complexes

Fig. 5. Effective atomic number versus photon energy for Dy(III)Cl(PhMal). $5 \mathrm{H}_{2} \mathrm{O}$

Fig. 6. Electron density versus photon energy for $\mathrm{Dy}(\mathrm{III}) \mathrm{Cl}(\mathrm{PhMal}) .5 \mathrm{H}_{2} \mathrm{O}$ 
Table 1. Some chemical parameters for Malonic and Phenylmalonic acids obtained with DFT/B3LYP method using SDD basis set

\begin{tabular}{lcc}
\hline Parameter $(\mathrm{eV})$ & Malonic acid & Phenylmalonic acid \\
\hline Energy Gap $(\Delta \mathrm{E})$ & 7.16 & 5.78 \\
Ionization energy $(\mathrm{I})$ & 8.24 & 7.01 \\
Electron affinity $(\mathrm{A})$ & 1.07 & 1.23 \\
Hardness $(\eta)$ & 3.58 & 2.89 \\
Softness $(\mathrm{S})$ & 0.28 & 0.35 \\
Chemical potential $(\mu)$ & -4.65 & -4.12 \\
Electronegativity $(\chi)$ & 4.65 & 4.12 \\
Global electrophilicity $(\omega)$ & 3.02 & 5.87 \\
\hline
\end{tabular}


Table 2. Experimental and theoretical values of total mass attenuation coefficient $\left(\mathrm{cm}^{2} / \mathrm{g}\right)$

\begin{tabular}{|c|c|c|c|c|c|c|c|c|c|c|c|c|c|c|c|}
\hline \multicolumn{16}{|c|}{ Total mass attenuation coefficient $(\mu / \rho)$} \\
\hline & \multicolumn{3}{|c|}{$13.92 \mathrm{keV}$} & \multicolumn{3}{|c|}{$17.75 \mathrm{keV}$} & \multicolumn{3}{|c|}{$20.78 \mathrm{keV}$} & \multicolumn{3}{|c|}{$26.34 \mathrm{keV}$} & \multicolumn{3}{|c|}{$59.54 \mathrm{keV}$} \\
\hline Sample & Exp. & WinX. & FFAST & Exp. & WinX. & FFAST & Exp. & WinX. & FFAST & Exp. & WinX. & FFAST & Exp. & WinX. & FFAST \\
\hline $\mathrm{Gd}$ & & 113.42 & 110.86 & & 59.76 & 58.28 & & 39.37 & 38.36 & $20.5 \pm 0.65$ & 20.93 & 20.69 & $12.32 \pm 0.13$ & 11.99 & 11.93 \\
\hline $\mathrm{Gd}(\mathrm{III}) \mathrm{Cl}_{3} \cdot 6 \mathrm{H}_{2} \mathrm{O}$ & & 54.92 & 53.67 & & 28.73 & 28.01 & & 18.85 & 18.38 & $9.98 \pm 0.16$ & 9.99 & 9.88 & $5.34 \pm 0.06$ & 5.26 & 5.23 \\
\hline $\mathrm{Gd}(\mathrm{III}) \mathrm{Cl}(\mathrm{Mal}) \cdot 5 \mathrm{H}_{2} \mathrm{O}$ & $46.93 \pm 1.08$ & 49.08 & 47.96 & $25.06 \pm 0.35$ & 25.80 & 25.16 & $17.58 \pm 0.29$ & 16.98 & 16.56 & $9.14 \pm 0.15$ & 9.05 & 8.95 & $4.99 \pm 0.06$ & 5.01 & 4.99 \\
\hline Dy & & 123.27 & 120.54 & & 65.19 & 63.83 & & 43.01 & 42.05 & $22.24 \pm 1.30$ & 22.91 & 22.68 & $12.57 \pm 0.13$ & 12.84 & 12.82 \\
\hline $\mathrm{Dy}(\mathrm{III}) \mathrm{Cl}_{3} \cdot 6 \mathrm{H}_{2} \mathrm{O}$ & & 59.98 & 58.63 & & 31.50 & 30.82 & $19.86 \pm 0.66$ & 20.71 & 20.24 & $11.02 \pm 0.18$ & 11.00 & 10.89 & $5.89 \pm 0.06$ & 5.72 & 5.71 \\
\hline $\mathrm{Dy}(\mathrm{III}) \mathrm{Cl}(\mathrm{Mal}) \cdot 5 \mathrm{H}_{2} \mathrm{O}$ & & 54.03 & 52.82 & $27.66 \pm 0.38$ & 28.50 & 27.90 & $18.78 \pm 0.30$ & 18.79 & 18.38 & $10.00 \pm 0.16$ & 10.03 & 9.93 & $5.36 \pm 0.06$ & 5.46 & 5.45 \\
\hline $\mathrm{Dy}(\mathrm{III}) \mathrm{Cl}(\mathrm{PhMal}) \cdot 5 \mathrm{H}_{2} \mathrm{O}$ & $44.79 \pm 0.72$ & 45.40 & 44.38 & $22.93 \pm 0.37$ & 23.96 & 23.45 & $15.08 \pm 0.60$ & 15.81 & 15.46 & $8.55 \pm 0.46$ & 8.45 & 8.36 & $4.51 \pm 0.13$ & 4.60 & 4.60 \\
\hline $\mathrm{Er}$ & & 133.92 & 131.38 & & 71.10 & 69.71 & & 46.99 & 46.07 & & 25.07 & 24.84 & $13.56 \pm 0.15$ & 13.88 & 13.74 \\
\hline $\mathrm{Er}(\mathrm{III}) \mathrm{Cl}_{3} \cdot 6 \mathrm{H}_{2} \mathrm{O}$ & & 65.44 & 64.16 & & 34.51 & 33.81 & & 22.73 & 22.28 & $12.03 \pm 0.20$ & 12.09 & 11.98 & $6.34 \pm 0.07$ & 6.27 & 6.20 \\
\hline $\mathrm{Er}(\mathrm{III}) \mathrm{Cl}(\mathrm{Mal}) \cdot 5 \mathrm{H}_{2} \mathrm{O}$ & $56.75 \pm 1.16$ & 59.35 & 58.56 & $30.79 \pm 0.42$ & 31.43 & 31.01 & $20.21 \pm 0.36$ & 20.76 & 20.47 & $10.81 \pm 0.16$ & 11.09 & 11.04 & $5.89 \pm 0.07$ & 5.99 & 5.98 \\
\hline $\mathrm{Er}(\mathrm{III}) \mathrm{Cl}(\mathrm{PhMal}) \cdot 5 \mathrm{H}_{2} \mathrm{O}$ & $48.51 \pm 0.87$ & 49.95 & 48.98 & $25.70 \pm 0.33$ & 26.46 & 25.94 & $17.30 \pm 0.34$ & 17.49 & 17.14 & $9.16 \pm 0.16$ & 9.35 & 9.27 & $5.00 \pm 0.06$ & 5.05 & 5.01 \\
\hline $\mathrm{Yb}$ & & 144.26 & 142.10 & & 76.81 & 75.38 & & 50.85 & 49.97 & & 27.19 & 26.96 & $3.16 \pm 0.03$ & 3.21 & 3.13 \\
\hline $\mathrm{Yb}(\mathrm{III}) \mathrm{Cl}_{3} \cdot 6 \mathrm{H}_{2} \mathrm{O}$ & & 71.08 & 69.95 & & 37.61 & 36.88 & $25.83 \pm 1.27$ & 24.82 & 24.37 & $13.42 \pm 0.26$ & 13.23 & 13.12 & $1.65 \pm 0.02$ & 1.61 & 1.58 \\
\hline $\mathrm{Yb}(\mathrm{IIII}) \mathrm{Cl}(\mathrm{Mal}) \cdot 5 \mathrm{H}_{2} \mathrm{O}$ & & 64.86 & 63.86 & $33.49 \pm 0.95$ & 34.46 & 33.81 & $21.75 \pm 0.53$ & 22.80 & 22.40 & $11.66 \pm 0.20$ & 12.20 & 12.10 & $1.52 \pm 0.02$ & 1.51 & 1.48 \\
\hline $\mathrm{Yb}(\mathrm{III}) \mathrm{Cl}(\mathrm{PhMal}) \cdot 5 \mathrm{H}_{2} \mathrm{O}$ & $53.59 \pm 1.22$ & 54.70 & 53.85 & $27.71 \pm 0.36$ & 29.07 & 28.52 & $19.39 \pm 0.32$ & 19.24 & 18.90 & $9.91 \pm 0.17$ & 10.31 & 10.23 & $1.29 \pm 0.03$ & 1.30 & 1.28 \\
\hline
\end{tabular}


Table 3. Experimental and theoretical values of effective atomic number

\begin{tabular}{|c|c|c|c|c|c|c|c|c|c|c|c|c|c|c|c|}
\hline \multicolumn{16}{|c|}{ Effective atomic number $\left(\mathrm{Z}_{\text {eff }}\right)$} \\
\hline & & $2 \mathrm{keV}$ & & & $75 \mathrm{keV}$ & & & $8 \mathrm{keV}$ & & & $4 \mathrm{keV}$ & & & $54 \mathrm{keV}$ & \\
\hline Sample & Exp. & WinX. & FFAST & Exp. & WinX. & FFAST & Exp. & WinX. & FFAST & Exp. & WinX. & FFAST & Exp. & WinX. & FFAST \\
\hline $\mathrm{Gd}$ & & 64.00 & 64.00 & & 64.00 & 64.00 & & 64.00 & 64.00 & $62.21 \pm 1.98$ & 64.00 & 64.00 & $65.77 \pm 0.67$ & 64.00 & 64.00 \\
\hline $\mathrm{Gd}(\mathrm{IIII}) \mathrm{Cl}_{3} \cdot 6 \mathrm{H}_{2} \mathrm{O}$ & & 44.16 & 44.16 & & 44.34 & 44.29 & & 44.18 & 44.02 & $43.19 \pm 0.70$ & 43.25 & 43.02 & $49.76 \pm 0.51$ & 49.00 & 48.73 \\
\hline $\mathrm{Gd}(\mathrm{III}) \mathrm{Cl}(\mathrm{Mal}) \cdot 5 \mathrm{H}_{2} \mathrm{O}$ & $46.90 \pm 1.08$ & 49.05 & 49.06 & $47.37 \pm 0.66$ & 48.75 & 48.63 & $49.76 \pm 0.83$ & 48.08 & 47.81 & $46.47 \pm 0.75$ & 46.01 & 45.64 & $47.57 \pm 0.53$ & 47.79 & 47.48 \\
\hline Dy & & 66.00 & 66.00 & & 66.00 & 66.00 & & 66.00 & 66.00 & $64.07 \pm 3.74$ & 66.00 & 66.00 & $64.62 \pm 0.67$ & 66.00 & 66.00 \\
\hline $\mathrm{Dy}(\mathrm{III}) \mathrm{Cl}_{3} \cdot 6 \mathrm{H}_{2} \mathrm{O}$ & & 47.71 & 47.72 & & 47.98 & 47.97 & $45.87 \pm 1.53$ & 47.84 & 47.71 & $46.97 \pm 0.77$ & 46.90 & 46.67 & $54.60 \pm 0.56$ & 53.05 & 52.82 \\
\hline Dy(III)Cl(Mal) $\cdot 5 \mathrm{H}_{2} \mathrm{O}$ & & 52.74 & 52.76 & $50.93 \pm 0.71$ & 52.48 & 52.40 & $51.78 \pm 0.83$ & 51.80 & 51.56 & $49.55 \pm 0.77$ & 49.68 & 49.31 & $50.48 \pm 0.55$ & 51.40 & 51.10 \\
\hline $\mathrm{Dy}(\mathrm{III}) \mathrm{Cl}(\mathrm{PhMal}) \cdot 5 \mathrm{H}_{2} \mathrm{O}$ & $50.55 \pm 0.81$ & 51.24 & 51.26 & $48.56 \pm 0.78$ & 50.73 & 50.62 & $47.49 \pm 1.88$ & 49.77 & 49.48 & $47.59 \pm 2.55$ & 47.00 & 46.58 & $46.81 \pm 1.35$ & 47.80 & 47.48 \\
\hline Er & & 68.00 & 68.00 & & 68.00 & 68.00 & & 68.00 & 68.00 & & 68.00 & 68.00 & $66.45 \pm 0.74$ & 68.00 & 68.00 \\
\hline $\mathrm{Er}(\mathrm{III}) \mathrm{Cl}_{3} \cdot 6 \mathrm{H}_{2} \mathrm{O}$ & & 49.98 & 50.03 & & 50.30 & 50.31 & & 50.20 & 50.10 & $49.04 \pm 0.80$ & 49.29 & 49.07 & $56.08 \pm 0.58$ & 55.38 & 55.08 \\
\hline $\mathrm{Er}(\mathrm{III}) \mathrm{Cl}(\mathrm{Mal}) \cdot 5 \mathrm{H}_{2} \mathrm{O}$ & $52.68 \pm 1.08$ & 55.09 & 55.48 & $53.75 \pm 0.73$ & 54.87 & 55.16 & $52.80 \pm 0.93$ & 54.23 & 54.35 & $50.85 \pm 0.76$ & 52.16 & 52.01 & $52.93 \pm 0.60$ & 53.78 & 53.81 \\
\hline $\mathrm{Er}(\mathrm{III}) \mathrm{Cl}(\mathrm{PhMal}) \cdot 5 \mathrm{H}_{2} \mathrm{O}$ & $52.06 \pm 0.93$ & 53.61 & 53.66 & $51.62 \pm 0.67$ & 53.14 & 53.06 & $51.66 \pm 1.00$ & 52.22 & 51.97 & $48.44 \pm 0.83$ & 49.50 & 49.09 & $49.67 \pm 0.58$ & 50.20 & 49.77 \\
\hline $\mathrm{Yb}$ & & 70.00 & 70.00 & & 70.00 & 70.00 & & 70.00 & 70.00 & & 70.00 & 70.00 & $68.84 \pm 0.55$ & 70.00 & 70.00 \\
\hline $\mathrm{Yb}(\mathrm{III}) \mathrm{Cl}_{3} \cdot 6 \mathrm{H}_{2} \mathrm{O}$ & & 52.26 & 52.36 & & 52.63 & 52.65 & $54.70 \pm 2.70$ & 52.55 & 52.49 & $52.42 \pm 1.03$ & 51.69 & 51.49 & $37.70 \pm 0.48$ & 36.84 & 36.12 \\
\hline $\mathrm{Yb}(\mathrm{III}) \mathrm{Cl}(\mathrm{Mal}) \cdot 5 \mathrm{H}_{2} \mathrm{O}$ & & 57.43 & 57.52 & $55.65 \pm 1.58$ & 57.25 & 57.20 & $54.03 \pm 1.32$ & 56.64 & 56.46 & $52.20 \pm 0.88$ & 54.63 & 54.29 & $34.07 \pm 0.46$ & 33.92 & 33.13 \\
\hline $\mathrm{Yb}(\mathrm{III}) \mathrm{Cl}(\mathrm{PhMal}) \cdot 5 \mathrm{H}_{2} \mathrm{O}$ & $54.83 \pm 1.25$ & 55.97 & 56.06 & $52.96 \pm 0.68$ & 55.55 & 55.47 & $55.08 \pm 0.91$ & 54.66 & 54.44 & $49.97 \pm 0.84$ & 52.00 & 51.60 & $28.89 \pm 0.68$ & 29.14 & 28.41 \\
\hline
\end{tabular}


Table 4. Experimental and theoretical values of effective electron density (electrons/g)

\begin{tabular}{|c|c|c|c|c|c|c|c|c|c|c|c|c|c|c|c|}
\hline \multicolumn{16}{|c|}{ Effective electron density $\left(\mathrm{N}_{\mathrm{E}}\right) \times 10^{24}$} \\
\hline & \multicolumn{3}{|c|}{$13.92 \mathrm{keV}$} & \multicolumn{3}{|c|}{$17.75 \mathrm{keV}$} & \multicolumn{3}{|c|}{$20.78 \mathrm{keV}$} & \multicolumn{3}{|c|}{$26.34 \mathrm{keV}$} & \multicolumn{3}{|c|}{$59.54 \mathrm{keV}$} \\
\hline Sample & Exp. & WinX. & FFAST & Exp. & WinX. & FFAST & Exp. & WinX. & FFAST & Exp. & WinX. & FFAST & Exp. & WinX. & FFAST \\
\hline $\mathrm{Gd}$ & & 0.25 & 0.25 & & 0.25 & 0.25 & & 0.25 & 0.25 & $0.24 \pm 0.01$ & 0.25 & 0.25 & $0.25 \pm 0.01$ & 0.25 & 0.25 \\
\hline $\mathrm{Gd}(\mathrm{III}) \mathrm{Cl}_{3} \cdot 6 \mathrm{H}_{2} \mathrm{O}$ & & 2.79 & 2.79 & & 2.80 & 2.80 & & 2.79 & 2.78 & $2.73 \pm 0.04$ & 2.73 & 2.72 & $3.14 \pm 0.03$ & 3.10 & 3.08 \\
\hline $\mathrm{Gd}(\mathrm{IIII}) \mathrm{Cl}(\mathrm{Mal}) \cdot 5 \mathrm{H}_{2} \mathrm{O}$ & $3.57 \pm 0.08$ & 3.73 & 3.73 & $3.60 \pm 0.05$ & 3.71 & 3.70 & $3.78 \pm 0.06$ & 3.66 & 3.64 & $3.53 \pm 0.06$ & 3.50 & 3.47 & $3.62 \pm 0.04$ & 3.63 & 3.61 \\
\hline Dy & & 0.24 & 0.24 & & 0.24 & 0.24 & & 0.24 & 0.24 & $0.24 \pm 0.01$ & 0.24 & 0.24 & $0.24 \pm 0.01$ & 0.24 & 0.24 \\
\hline $\mathrm{Dy}(\mathrm{III}) \mathrm{Cl}_{3} \cdot 6 \mathrm{H}_{2} \mathrm{O}$ & & 2.94 & 2.94 & & 2.96 & 2.96 & $2.83 \pm 0.09$ & 2.95 & 2.94 & $2.90 \pm 0.05$ & 2.89 & 2.88 & $3.36 \pm 0.03$ & 3.27 & 3.26 \\
\hline $\mathrm{Dy}(\mathrm{III}) \mathrm{Cl}(\mathrm{Mal}) \cdot 5 \mathrm{H}_{2} \mathrm{O}$ & & 3.92 & 3.92 & $3.78 \pm 0.05$ & 3.90 & 3.89 & $3.85 \pm 0.06$ & 3.85 & 3.83 & $3.68 \pm 0.06$ & 3.69 & 3.66 & $3.75 \pm 0.04$ & 3.82 & 3.80 \\
\hline $\mathrm{Dy}(\mathrm{III}) \mathrm{Cl}(\mathrm{PhMal}) \cdot 5 \mathrm{H}_{2} \mathrm{O}$ & $5.10 \pm 0.08$ & 5.17 & 5.17 & $4.90 \pm 0.08$ & 5.11 & 5.10 & $4.79 \pm 0.19$ & 5.02 & 4.99 & $4.80 \pm 0.26$ & 4.74 & 4.70 & $4.72 \pm 0.14$ & 4.82 & 4.79 \\
\hline Er & & 0.24 & 0.24 & & 0.24 & 0.24 & & 0.24 & 0.24 & & 0.24 & 0.24 & $0.24 \pm 0.01$ & 0.24 & 0.24 \\
\hline $\mathrm{Er}(\mathrm{III}) \mathrm{Cl}_{3} \cdot 6 \mathrm{H}_{2} \mathrm{O}$ & & 3.01 & 3.02 & & 3.03 & 3.03 & & 3.03 & 3.02 & $2.96 \pm 0.05$ & 2.97 & 2.96 & $3.38 \pm 0.03$ & 3.34 & 3.32 \\
\hline $\mathrm{Er}(\mathrm{III}) \mathrm{Cl}(\mathrm{Mal}) \cdot 5 \mathrm{H}_{2} \mathrm{O}$ & $3.83 \pm 0.08$ & 4.01 & 4.04 & $3.91 \pm 0.05$ & 3.99 & 4.01 & $3.84 \pm 0.07$ & 3.95 & 3.95 & $3.70 \pm 0.06$ & 3.80 & 3.78 & $3.85 \pm 0.04$ & 3.91 & 3.92 \\
\hline $\mathrm{Er}(\mathrm{IIII}) \mathrm{Cl}(\mathrm{PhMal}) \cdot 5 \mathrm{H}_{2} \mathrm{O}$ & $5.14 \pm 0.09$ & 5.29 & 5.30 & $5.10 \pm 0.07$ & 5.25 & 5.24 & $5.10 \pm 0.10$ & 5.16 & 5.13 & $4.78 \pm 0.08$ & 4.89 & 4.85 & $4.91 \pm 0.06$ & 4.96 & 4.92 \\
\hline $\mathrm{Yb}$ & & 0.22 & 0.22 & & 0.22 & 0.22 & & 0.22 & 0.22 & & 0.22 & 0.22 & $0.22 \pm 0.01$ & 0.22 & 0.22 \\
\hline $\mathrm{Yb}(\mathrm{III}) \mathrm{Cl}_{3} \cdot 6 \mathrm{H}_{2} \mathrm{O}$ & & 3.07 & 3.08 & & 3.09 & 3.09 & $3.21 \pm 0.16$ & 3.09 & 3.08 & $3.08 \pm 0.06$ & 3.04 & 3.02 & $2.21 \pm 0.03$ & 2.16 & 2.12 \\
\hline $\mathrm{Yb}(\mathrm{IIII}) \mathrm{Cl}(\mathrm{Mal}) \cdot 5 \mathrm{H}_{2} \mathrm{O}$ & & 4.08 & 4.08 & $3.95 \pm 0.11$ & 4.06 & 4.06 & $3.84 \pm 0.09$ & 4.02 & 4.01 & $3.71 \pm 0.06$ & 3.88 & 3.85 & $2.42 \pm 0.03$ & 2.41 & 2.35 \\
\hline $\mathrm{Yb}(\mathrm{III}) \mathrm{Cl}(\mathrm{PhMal}) \cdot 5 \mathrm{H}_{2} \mathrm{O}$ & $5.28 \pm 0.12$ & 5.39 & 5.40 & $5.10 \pm 0.07$ & 5.35 & 5.34 & $5.31 \pm 0.09$ & 5.27 & 5.25 & $4.81 \pm 0.08$ & 5.01 & 4.97 & $2.78 \pm 0.07$ & 2.81 & 2.74 \\
\hline
\end{tabular}


Page 17 of 22

Canadian Journal of Physics

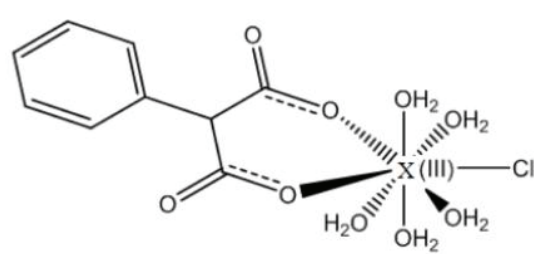

(a)

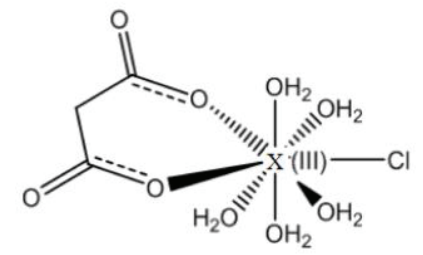

(b)

Fig. 1. (abb)

https://mc06.manuscriptcentral.com/cjp-pubs 


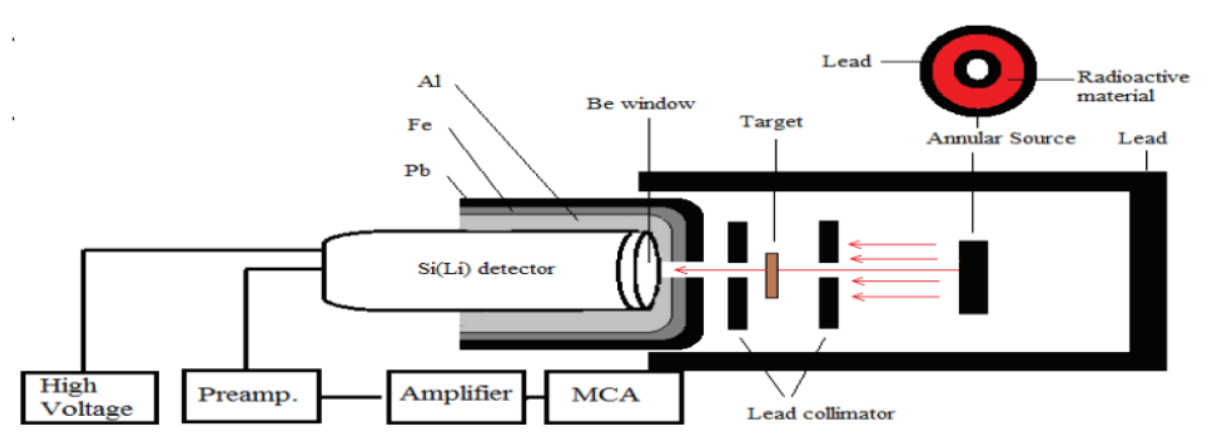

Fig. 2. 


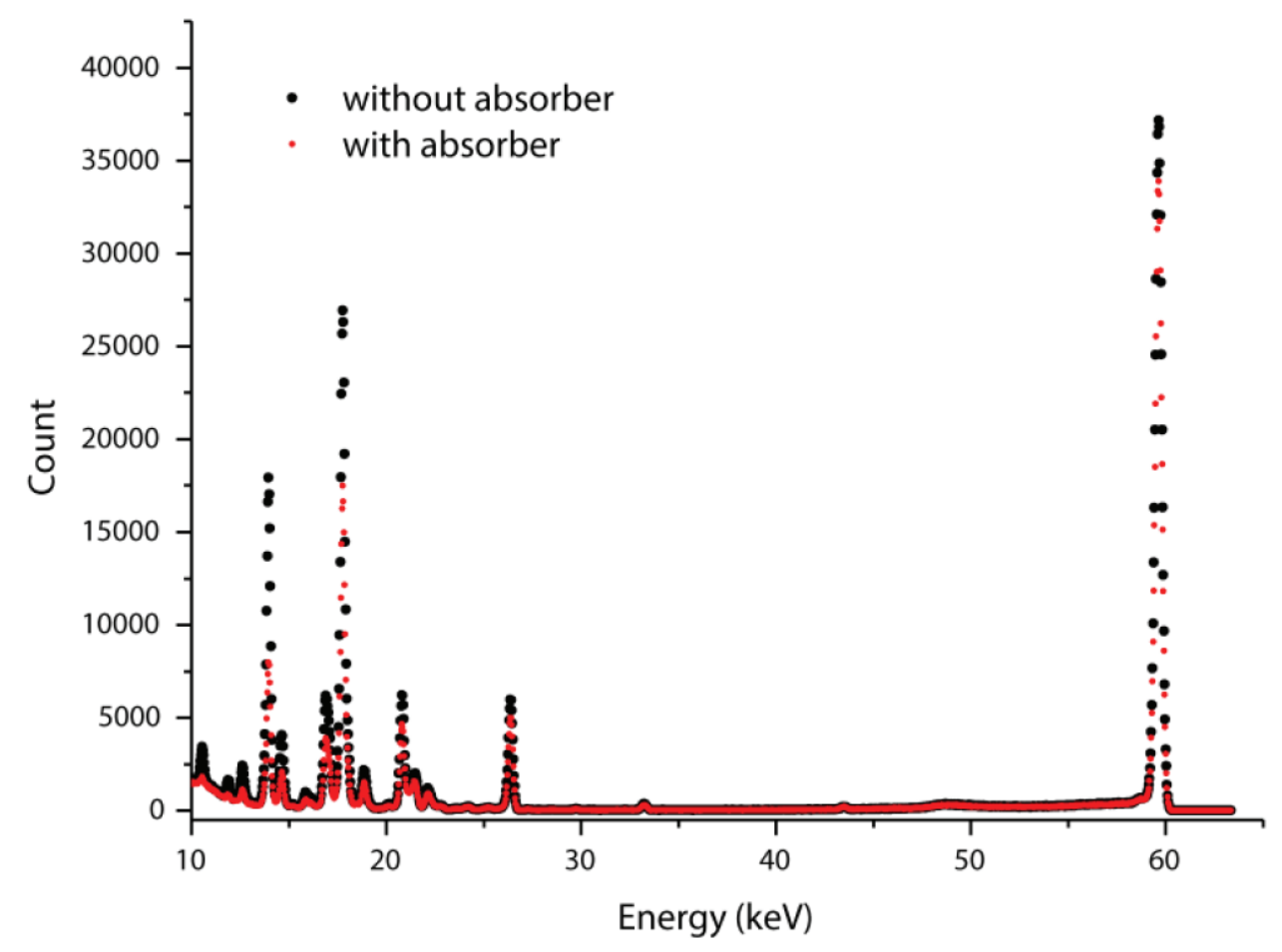

Fig. 3. 


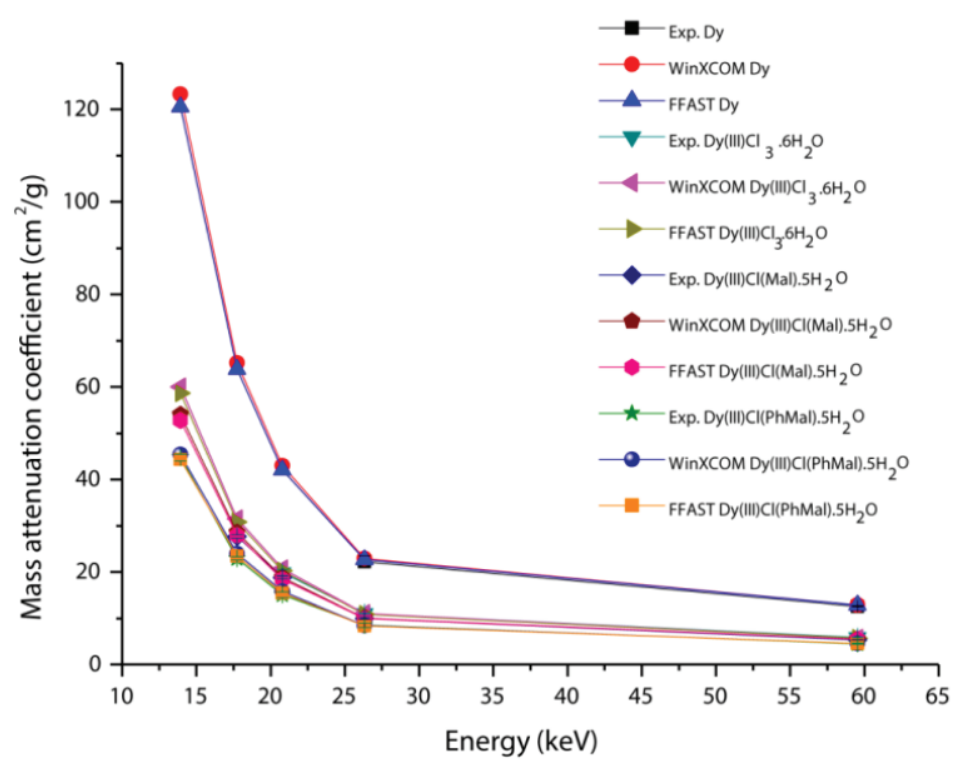

Fig. 4 


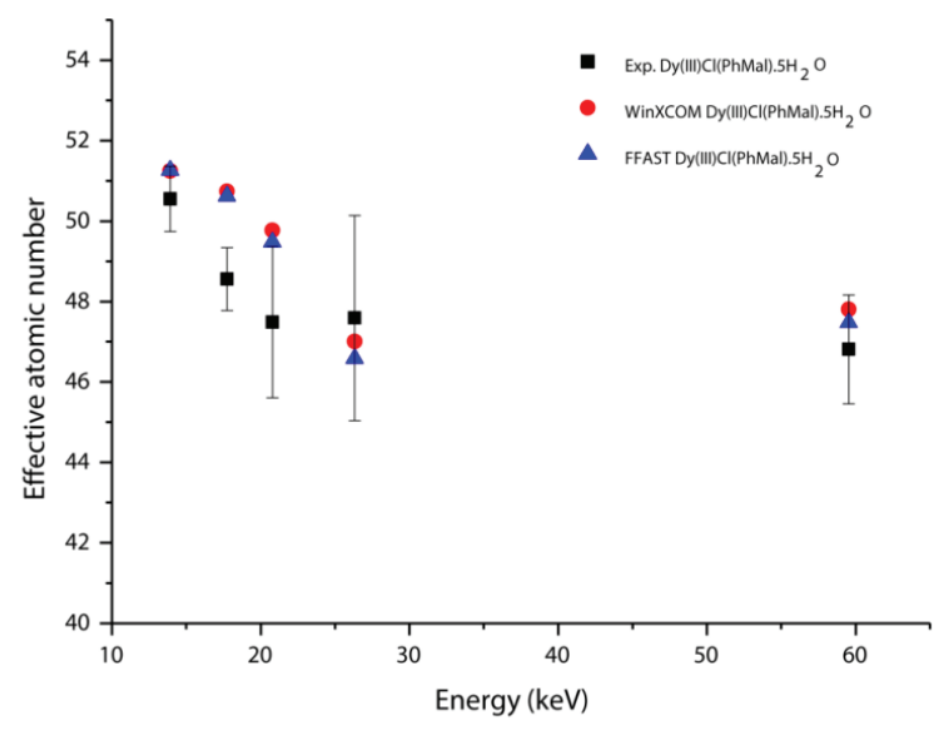

Fig. 5 


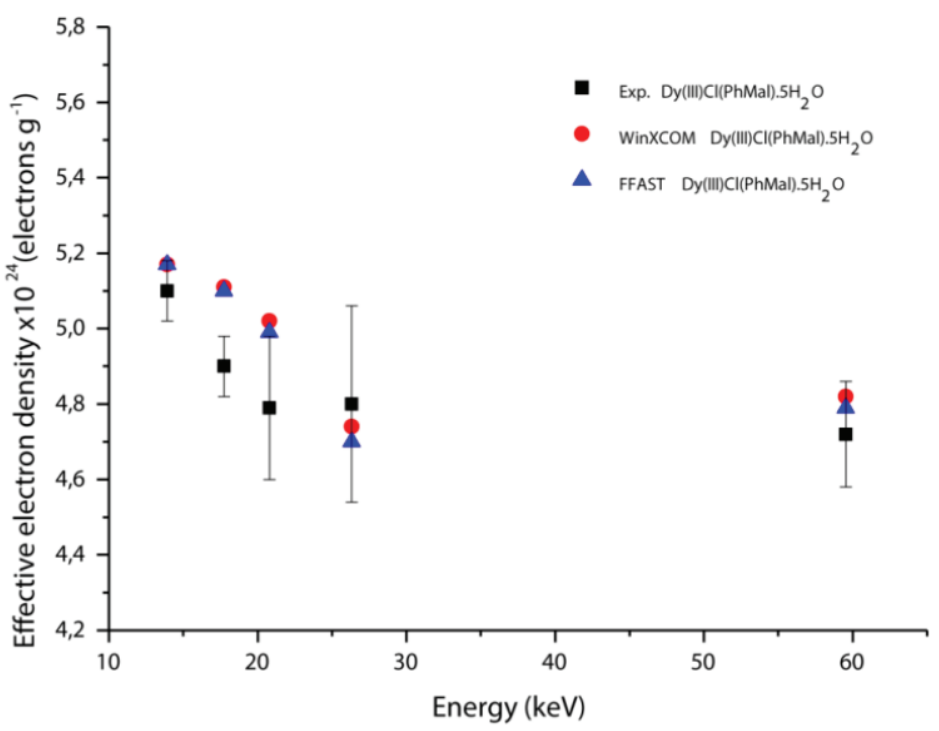

Fig. 6 